\title{
Sorption of polycyclic aromatic hydrocarbons on particulate organic matters
}

\author{
Xueyan Guo ${ }^{\mathrm{a}, \mathrm{b}}$, Lei Luo ${ }^{\mathrm{a}}$, Yibing Mab ${ }^{\mathrm{b}}$, Shuzhen Zhang ${ }^{\mathrm{a}, *}$ \\ a State Key Laboratory of Environmental Chemistry and Ecotoxicology, Research Center for Eco-Environmental Sciences, Chinese Academy of Sciences, \\ P. O. Box 2871, Beijing 100085, China \\ ${ }^{\mathrm{b}}$ Ministry of Agriculture Key Laboratory of Plant Nutrition and Nutrient Cycling, Institute of Agricultural Resources and Regional Planning, \\ Chinese Academy of Agricultural Sciences, Beijing 100081, China
}

\section{A R T I C L E I N F O}

\section{Article history:}

Received 9 January 2009

Received in revised form 12 August 2009

Accepted 14 August 2009

Available online 22 August 2009

\section{Keywords:}

Sorption

Desorption

Particulate organic matter

Polycyclic aromatic hydrocarbons

\begin{abstract}
A B S T R A C T
Particulate organic matter (POM) is a key organic matter fraction which can influence soil fertility. Its interactions with hydrophobic organic pollutants (HOCs) have not been characterized and the mechanisms of retention of HOCs by POM remain unclear. In the present study, sorption behaviors of polycyclic aromatic hydrocarbons (PAHs) naphthalene (NAP), phenanthrene (PHE), and pyrene (PYR) by POMs separated from different soils were examined and the POMs were characterized by elemental analysis, solid state ${ }^{13} \mathrm{C}$ NMR, and Fourier transform infrared spectroscopy (FT-IR). The results indicated that POMs were mainly composed of aliphatic components with high polarity. The different original POMs showed similar chemical composition and configuration. Sorption behaviors of PAHs indicated that there was no significant difference in sorption capacity among the POMs. Sorption of NAP and PHE by POMs displayed a nonlinear isotherm, while sorption of PYR yielded a linear isotherm. No significant hysteresis and ionic strength effect were observed for PAH desorption from the POMs.
\end{abstract}

(C) 2009 Elsevier B.V. All rights reserved.

\section{Introduction}

Sorption of hydrophobic organic contaminants (HOCs) on solid matrices such as soils and sediments is one of the key processes which determine the fate of contaminants in the environment $[1,2]$. It has been recognized that various forms of soil organic matter (SOM) serve as dominant environmental "compartments" for sorption and accumulation of HOCs and determine their fate in soils. Therefore, intensive studies have been carried out to characterize sorption of HOCs on different SOM fractions and to elucidate the relevant sorption mechanisms [3-8].

Soil organic matter is a complex, heterogeneous material. In addition to the well-known humic fractions such as humic acid, fulvic acid, and humin, there exists a large quantity of particulate organic matter (POM) which consists of light-density, sand-sized $(>0.05 \mathrm{~mm})$ organic debris with a relatively rapid turnover. POM is more likely to be free of binding to other soil components such as soil minerals although some of POMs can be occluded within microaggregates [9]. POM is considered as a key organic matter fraction which influences soil fertility and has the potential to be used as an indicator of soil quality [10]. As an important SOM fraction particularly accumulating at soil surface, interaction of POM with soil pollutants is very important. But until now only

\footnotetext{
* Corresponding author. Tel.: +86 10 62849683; fax: +8610 62923563

E-mail address: szzhang@rcees.ac.cn (S. Zhang).
}

interactions between POM and heavy metals [11-14] and some pesticides $[15,16]$ have been studied. Its interaction with HOCs remains uninvestigated. POM has a heterogeneous composition and its composition and characteristics may be related to its origin and humification history and soil property as well. Therefore, we hypothesized there should be differences in sorption of HOCs on POMs which originate from different soils under different land uses.

Thus, the objective of this work was to characterize and quantify sorption and desorption of PAHs on four kinds of POMs separated from the soils which were sampled from different sites and had different organic inputs. Elemental analysis, Fourier transform infrared (FT-IR) spectroscopy and solid-state ${ }^{13} \mathrm{C}$ NMR spectra were employed to characterize the POMs with the aim to compare the characteristics of POMs with different origins and to elucidate the relevant mechanisms for sorption of PAHs on POM.

\section{Materials and methods}

\subsection{Sorbates and sorbents}

As model HOCs, three PAHs, naphthalene (NAP), phenanthrene (PHE), and pyrene (PYR) with different $\log K_{\text {ow }}$ value and aqueous solubility were chosen as sorbates. They were purchased from Sigma-Aldrich Chemical Co. and used without further purification. Selected properties of the PAHs are listed in Table 1. 
Table 1

Selected properties of $\mathrm{PAHs}^{\mathrm{a}}[19]$

\begin{tabular}{llccllc}
\hline PAHs & $M$ & MP & $S_{\mathrm{w}}$ & $\log K_{\text {ow }}$ & $\Delta H$ & $S_{\mathrm{s}}$ \\
\hline NAP & 128.2 & 80 & 31.5 & 3.36 & 4.61 & 106.6 \\
PHE & 178.2 & 101 & 1.29 & 4.57 & 4.45 & 5.9 \\
PYR & 202.3 & 156 & 0.135 & 5.18 & 4.2 & 1.18 \\
\hline
\end{tabular}

a $M$ : molecular weight, g/mol. MP: melting point, ${ }^{\circ} \mathrm{C} . S_{\mathrm{w}}$ : aqueous solubility, $\mathrm{mg} / \mathrm{L}$ $K_{\text {ow }}$ : octanol-water partition coefficient. $\Delta H$ : heat of fusion, kcal $/ \mathrm{mol}$. $S_{\mathrm{s}}$ : the supercooled liquid-state solubility, $\mathrm{mg} / \mathrm{L}$.

Four soil samples were collected from the surface (depth, $0-15 \mathrm{~cm}$ ) of plantation fields of tree-of-heaven (Ailanthus altissima) (Soil1) and pine (Pinus massoniana) (Soil2) in a dark brown soil in Beijing and wheat (Triticum aestivum) in the dark brown soil in Beijing (Soil3) and a black chernozem soil in Heilongjiang (Soil4). The $\mathrm{pH}$ values of the soils with a soil-to-water ratio of $1: 2(\mathrm{w} / \mathrm{v})$ were $7.5,6.9,6.9,7.4$ and the SOM contents were $11.7 \%, 14.6 \%, 3.38 \%$ and $6.86 \%$ for Soil1, Soil2, Soil3 and Soil4, respectively. Tree plantation resulted in a higher SOM contents in the surface soils. These soils were sampled for POM separation with the aim to represent different soil organic matter contents and different POM origins and degree of humification. The corresponding POMs were labeled as POM1, POM2, POM3 and POM4, respectively. The soil samples were air-dried, ground, and passed through a 2-mm nylon sieve. Physical fractionation of the POMs was then achieved using the method described by Ducaroir and Lamy [17] and Guo et al. [13]. In brief, the soils were agitated in deionized water $(500 \mathrm{~g} / \mathrm{L})$ with glass beads by shaking for $16 \mathrm{~h}$, leading to the complete dispersion. Suspensions were then passed successively through a $0.2-$ and $0.05-\mathrm{mm}$ sieve to obtain soil fractions in $0.05-0.2 \mathrm{~mm}$ and $>0.2 \mathrm{~mm}$. POM in both soil fractions was separated separately by repeated flotationpanning in deionized water and oven-dried at $55^{\circ} \mathrm{C}$ for $48 \mathrm{~h}$. The POM in fraction $>0.2 \mathrm{~mm}$ accounted $70 \%, 68 \%, 59 \%$ and $54 \%$ of the total POM for POM1, POM2, POM3 and POM4. It was ground to pass a $0.2-\mathrm{mm}$ standard sieve and then combined with the $0.05-0.2 \mathrm{~mm}$ fraction, which was then ready for the use in the following experiments. The whole fractions of POM represented 4.2\%, 2.8\%, 0.58\% and $0.92 \%$ of the bulk soils (w/w) for POM1, POM2, POM3 and POM4, respectively.

\subsection{Characterization of sorbents}

The total contents of $\mathrm{C}, \mathrm{H}$ and $\mathrm{N}$ in the POMs and soils were determined using a CHN Elemental Analyzer (Vario EL, Elementar Analysensyteme, Hanau, Germany), and the ash content was determined by combustion of the POM at $750^{\circ} \mathrm{C}$ for $4 \mathrm{~h}$. Oxygen content was calculated by mass difference. Infrared spectra were obtained using a GX2000 FT-IR spectrophotometer (PerkinElmer) equipped with deuterated triglycine and mercury-cadmium-telluride detectors (Thermo Nicolet, Madison, USA), a KBr beam splitter (Thermo Nicolet), and a sample bench purged with dry air. The resolution for the FT-IR spectra was $4.0 \mathrm{~cm}^{-1}$, and a total of 32 scans were collected for each spectrum. The FT-IR spectra were recorded with pellets obtained by pressing a mixture of $1 \mathrm{mg}$ of sample and $100 \mathrm{mg}$ of $\mathrm{KBr}$ under reduced pressure. Solid-state ${ }^{13} \mathrm{C}$ NMR spectra were obtained using a Bruker DSX-300 spectrometer (Karlsruhe, Germany) operated at ${ }^{13} \mathrm{C}$ frequency of $300 \mathrm{MHz}$. Additional parameters used to acquire and process the spectra are as follows: contact time of $2 \mathrm{~ms}$; recycle delay time of $1 \mathrm{~s}$; line broadening of $15 \mathrm{~Hz}$; spinning speed of $13 \mathrm{kHz}$ and the number of scans was from 5000 to 10,000 per sample.

\subsection{Sorption and desorption isotherms}

All sorption isotherms were obtained using a batch equilibration technique in $50 \mathrm{~mL}$ glass centrifuge tubes sealed by Teflon-lined screw caps at room temperature $\left(20 \pm 1{ }^{\circ} \mathrm{C}\right)$. The background solution was comprised of $0.005 \mathrm{~mol} / \mathrm{L} \mathrm{CaCl}_{2}$ in deionized water with $100 \mathrm{mg} / \mathrm{L} \mathrm{NaN}_{3}$ as a biocide. Initial concentrations ranged from 0.15 to $15 \mathrm{mg} / \mathrm{L}$ for NAP, 0.01 to $1.0 \mathrm{mg} / \mathrm{L}$ for PHE, and 0.0048 to $0.08 \mathrm{mg} / \mathrm{L}$ for PYR. Due to the low aqueous solubility, stock solutions were made in methanol before being added to the background solution. The concentration of methanol in the final solutions was always maintained below $0.5 \%(\mathrm{v} / \mathrm{v})$ to minimize co-solvent effects. Sorption of the PAHs can reach equilibrium after $3 \mathrm{~d}$ for the concentration range in this study base on the preliminary experiments (data not shown). The solid-to-liquid ratio was varied for different compounds and POM in order to achieve $30-80 \%$ of the equilibrium sorption. Isotherms consisted of 7-11 concentration points; each point, including the blank, was run in duplicate. The vials were sealed with aluminum-foil-lined Teflon screw caps and then placed on a shaker for $3 \mathrm{~d}$ at room temperature $\left(20 \pm 1^{\circ} \mathrm{C}\right)$. The solutions with different $\mathrm{CaCl}_{2}$ concentrations $(0.005$ or $0.05 \mathrm{~mol} / \mathrm{L})$ and $100 \mathrm{mg} / \mathrm{L} \mathrm{NaN}_{3}$, and PHE were also used as background solutions and a model pollutant, respectively, to examine the effect of ionic strength on PAH sorption. After a 3-d equilibration period, the samples were centrifuged for $30 \mathrm{~min}$ at $2000 \times \mathrm{g}$, and supernatants were sampled for high-performance liquid chromatography (HPLC) analysis.

Desorption of PAHs from POM was conducted by a single decant-refill cycle technique after the completion of the sorption experiments [18]. After the $1-\mathrm{mL}$ aliquot was withdrawn, as part of the sorption procedure, the supernatant was further withdrawn to discard $50 \%$ of the whole supernatant and replaced by the fresh background solution (dilution). Dilution factors (80\%) for the different concentrations were determined by weight. Following the dilution, the vials were mixed for the same equilibration time of $3 \mathrm{~d}$ as used in the sorption experiments. In conducting sorption and desorption experiments, control of the mass balance was always investigated. Control experiments with PAHs and without sorbent were conducted as sorption-desorption steps. The recoveries of PAHs are in the range of $97-102 \%$, suggesting that the losses of PAHs by evaporation, bio- and photo-degradation, or sorption to glass vial are negligible. A major concern in the organic compound desorption experiments is the loss of sorbent during supernatant decanting. Therefore, to eliminate experimental error, the dry weights of all sorbents were determined before and after the sorption and desorption experiments. No significant change in the sorbent dry weights was observed. The final extraction of the solids after desorption by methanol was further conducted to check the mass balance. As a result, no mass loss was observed in the whole sorption-desorption processes.

Concentrations of PAHs were determined using a reversedphase HPLC ( $\mathrm{C}_{18}$ column) with a UV-vis detector at an absorption wavelength of $254 \mathrm{~nm}$ for NAP and PHE and a fluorescence detector at the excitation/emission wavelength of 260/420 nm for PYR. The mobile phase was methanol: water (85:15 for NAP; 90:10 for PHE; 95:5 for PYR) with a flow rate of $1.0 \mathrm{~mL} \mathrm{~min}^{-1}$. Under the experimental conditions described, NAP, PHE and PYR showed a single HPLC peak at the retention time of 4.5, 6.5, 6.0 min, respectively. The solid-phase sorbate PAH concentrations were calculated for each reactor from a solute mass balance between the two phases.

\subsection{Data analysis}

Sorption of HOCs by heterogeneous sorbents is often described by the Freundlich equation $\left(q_{\mathrm{e}}=K_{\mathrm{f}} C_{\mathrm{e}}^{n}\right)$, where $q_{\mathrm{e}}$ is the amount of sorbate sorbed per unit mass of sorbent $(\mathrm{mg} / \mathrm{kg}), C_{\mathrm{e}}$ is the concentration of sorbate in equilibrium solution $(\mathrm{mg} / \mathrm{L}), K_{\mathrm{f}}$ is the Freundlich sorption coefficient $\left[(\mathrm{mg} / \mathrm{kg}) /(\mathrm{mg} / \mathrm{L})^{-n}\right]$ which provides an index of sorption capacity, $n$ is the isotherm nonlinearity parameter, an indicator of site energy heterogeneity. To compare the 
Table 2

Elemental compositions, ash contents and atomic ratios of different original POMs ${ }^{\mathrm{a}}$.

\begin{tabular}{|c|c|c|c|c|c|c|c|}
\hline \multirow[t]{2}{*}{ Sample } & \multicolumn{3}{|c|}{$\begin{array}{l}\text { Elemental } \\
\text { composition (wt.\%) }\end{array}$} & \multicolumn{3}{|c|}{ Atomic ratio } & \multirow[t]{2}{*}{ Ash (wt.\%) } \\
\hline & $\mathrm{C}$ & $\mathrm{H}$ & $\mathrm{N}$ & $\mathrm{C} / \mathrm{N}$ & $\mathrm{H} / \mathrm{C}$ & $(\mathrm{O}+\mathrm{N}) / \mathrm{C}^{\mathrm{b}}$ & \\
\hline POM1 & 48.5 & 6.8 & 2.4 & 23.56 & 1.68 & 0.70 & 28.5 \\
\hline POM2 & 46.8 & 6.7 & 2.4 & 22.80 & 1.71 & 0.75 & 21.9 \\
\hline РОM3 & 48.6 & 6.2 & 3.3 & 17.28 & 1.53 & 0.70 & 25.8 \\
\hline POM4 & 44.8 & 6.8 & 3.4 & 15.38 & 1.83 & 0.82 & 30.2 \\
\hline
\end{tabular}

a Values are on an ash-free basis.

b Oxygen content was calculated by the mass difference.

sorption characteristics of different sorbents directly, a modified Freundlich parameter, $K_{\mathrm{f}}^{\prime}(\mathrm{mg} / \mathrm{kg})$, was calculated after normalizing $C_{\mathrm{e}}$ by the supercooled liquid-state solubility $\left(S_{\mathrm{s}}\right)$ of PAHs. $q_{\mathrm{e}}=K_{\mathrm{f}}^{\prime} C_{\mathrm{r}}^{n}$, where $C_{\mathrm{r}}$ is the reduced concentration, i.e., solubilitynormalized equilibrium concentration) [19]. The values of $K_{\mathrm{f}}^{\prime}$ and $n$ for all sorption isotherms were calculated by Freundlich equation fit using Origin 8.0 at the $95 \%$ confidence level. Statistical analysis of the results was performed in SPSS (version 11.5, SPSS Inc.) using ANOVAs (Tukey test, $p<0.05$ ).

\section{Results and discussion}

\subsection{Characterization of POM}

\subsubsection{Elemental compositions of POM}

The elemental compositions of the isolated POMs are presented in Table 2. Lower $\mathrm{C} / \mathrm{N}$ ratio suggests a greater humification [20], and therefore the humification degrees of the POMs were in the following order: POM4 > POM3 > POM2 > POM1. The ash content in POMs was high, especially in POM4, which was most likely due to the formation of organic-inorganic complexes during humification [21]. The organic carbon content of POM1 was close to that of POM3, and higher than that of POM2 and POM4. The lowest organic carbon content of POM4 (44.8\%) probably correlated to its highest degree of humification. The $\mathrm{H} / \mathrm{C}$ ratios were in the order: POM4 (1.83)> POM2 (1.71) > POM1 (1.68) $>$ POM3 (1.53). The polarity, here defined as the atomic ratio of $(\mathrm{N}+\mathrm{O}) / \mathrm{C}$ [22], ranged from 0.70 to 0.82 and changed slightly among the POMs. POM4 showed the highest $\mathrm{H} / \mathrm{C}$ ratio (1.83) and polarity (0.82). These elemental composition data indicate that the POMs are mainly aliphatic components with comparable polarities, which is in agreement with the following spectroscopic data.

\subsubsection{Analyses of Fourier transform infrared (FT-IR) spectra}

The POMs of different origins have similar FT-IR spectra (Fig. 1). The broad and strong bands observed at about $3410 \mathrm{~cm}^{-1}$ are dominated by stretching vibration of hydroxyl functional groups,

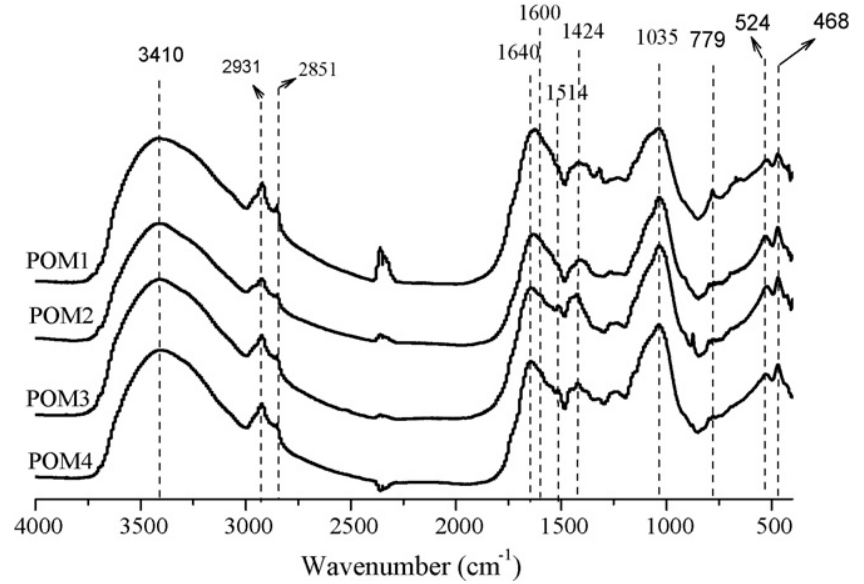

Fig. 1. Fourier transform infrared spectra of the POMs from different origins.

indicative of hydrogen bonding. The absence of absorption around $3600 \mathrm{~cm}^{-1}$ suggests that there are no appreciable free hydroxyl groups in POMs [23]. Peaks at 2931 and $2851 \mathrm{~cm}^{-1}$ are ascribed to aliphatic $\mathrm{CH}_{2}$ asymmetric and symmetric stretching, respectively. Absorption at about $1640 \mathrm{~cm}^{-1}$ is usually attributed to $\mathrm{C}=0$ stretching of amide groups, quinine $\mathrm{C}=\mathrm{O}$ and/or $\mathrm{C}=\mathrm{O}$ of $\mathrm{H}$-bonded conjugated ketones. The weak absorption bands at 1600, 1514 and $1424 \mathrm{~cm}^{-1}$ are assigned to aromatic ring carbon skeletal stretching. The strong peak that appears at about $1035 \mathrm{~cm}^{-1}$ results from $\mathrm{C}-\mathrm{O}$ stretching vibrations of polysaccharides, $\mathrm{Si}-\mathrm{O}$ vibrations of silicate impurity. These observations indicate that POMs are primarily dominated by polysaccharides and have low content of aromatic carbon [24].

\subsection{3. ${ }^{13}$ C NMR spectra of POMs}

The solid-state ${ }^{13} \mathrm{C}$ NMR spectra and integration results of the POMs are shown in Fig. 2 and Table 3. The ${ }^{13} \mathrm{C}$ NMR spectra of different original POMs demonstrate similar chemical shifts. According to the chemical shifts, seven regions can be used to reflect the composition of different types of carbon: alkyl-C (0-45 ppm), O-alkyl-C (45-109 ppm), nonpolar aromatic C (109-142 ppm), polar aromatic C (142-163 ppm), carboxylic- and amide-C (163-190 ppm); and ketonic/aldehyde-C (190-220 ppm). The percentage of polar organic carbon (POC) was calculated as the combined fractions of the 45-109 and 142-220 ppm regions of the NMR spectra $[25,26]$. Similar carbon distribution among POM2, POM3 and POM4 suggests that they have similar structures. According to the integration results from the solid-state ${ }^{13} \mathrm{C}$ NMR analysis, the content of aliphatic carbon (0-109 ppm) ranging 58.2-62.6\% in POM is far more than that of aromatic carbon (19.2-24.3\%). The aliphaticity of POM4 is the smallest, thus its aromaticity is the highest. The aliphaticity order, POM1 > POM2 > POM3 > POM4, is consistent with

Table 3

Integration results from solid-state ${ }^{13} \mathrm{C}$ NMR analysis ${ }^{\mathrm{a}}$ of different original POMs.

\begin{tabular}{|c|c|c|c|c|c|c|c|c|c|c|}
\hline \multirow[t]{2}{*}{ Sample } & \multicolumn{6}{|c|}{ Relative distribution of $C$ chemical shift $(\mathrm{ppm})(\%)^{\mathrm{b}}$} & \multirow[t]{2}{*}{ Aliphatic C } & \multirow[t]{2}{*}{ Aromatic C } & \multirow[t]{2}{*}{ Aliphaticity ${ }^{\mathrm{C}}$} & \multirow[t]{2}{*}{ POC (\%) } \\
\hline & $\begin{array}{l}\text { Alkyl C } \\
0-45\end{array}$ & $\begin{array}{l}\text { O-alkyl C } \\
45-109\end{array}$ & $\begin{array}{l}\text { Aromatic C } \\
109-142\end{array}$ & $\begin{array}{l}\text { O-aryl C } \\
142-163\end{array}$ & $\begin{array}{l}\text { Carboxyl C } \\
163-190\end{array}$ & $\begin{array}{l}\text { Carbonyl C } \\
190-220\end{array}$ & & & & \\
\hline POM1 & 26.4 & 34.5 & 12.8 & 6.4 & 17.9 & 2.1 & 60.9 & 19.2 & 3.2 & 60.8 \\
\hline POM2 & 19.8 & 42.8 & 12.3 & 10.2 & 11.6 & 3.3 & 62.6 & 22.5 & 2.8 & 67.8 \\
\hline POM3 & 18.9 & 43.2 & 15.6 & 8.0 & 10.1 & 4.2 & 62.1 & 23.6 & 2.6 & 65.5 \\
\hline POM4 & 18.9 & 39.3 & 14.6 & 9.7 & 12.5 & 5.0 & 58.2 & 24.3 & 2.4 & 66.5 \\
\hline
\end{tabular}

a Integration was performed on a relative basis rather than an absolute basis.

b Percentage of total ${ }^{13} \mathrm{C}$ NMR signal $(0-220 \mathrm{ppm})$.

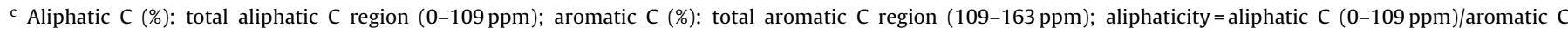

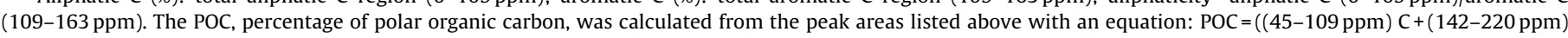
C) $/(0-220 \mathrm{ppm}) \mathrm{C} \times 100$. 


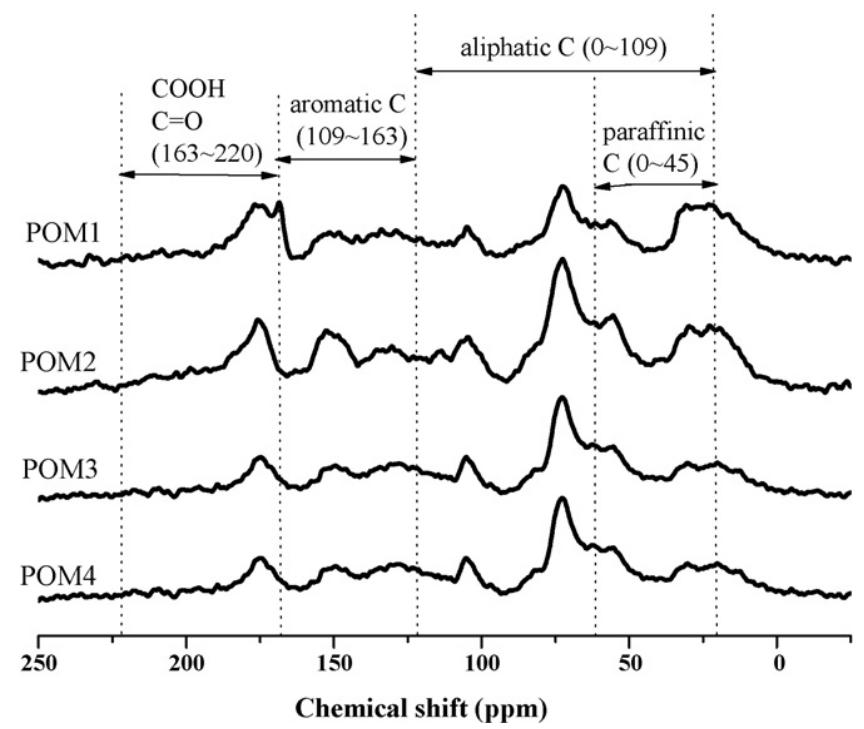

Fig. 2. Solid-state ${ }^{13} \mathrm{C}$ NMR spectra of the POMs from different origins.

the results obtained from elemental analysis, indicating that POM4 is the highest degree of humification among the POMs. POM is mainly composed of aliphatic carbon and contains several oxygen and nitrogen functional groups with higher polarity, which is consistent with the results of FT-IR spectra.

\subsection{Sorption and desorption isotherms}

Isotherms for NAP, PHE and PYR sorption on the different original POMs are presented in Fig. 3, which were well described by the Freundlich equation with the correlation coefficients higher than 0.992 (Table 4). The Freundlich equation parameters ( $K_{\mathrm{f}}^{\prime}$ and $n$ ) represent sorption affinity and isotherm nonlinearity, respectively. Weber et al. [27] have suggested that the $n$ value can be taken as an index of site energy distribution (i.e., the smaller the $n$, the more heterogeneous the sorption site). The $n$ values $(n>0.995)$ for POM1 were higher than those for POM2, POM3 and POM4 while the values for POM2, POM3 and POM4 did not differ greatly from each other $(p>0.05)$. Consequently, POM1 with relatively homogeneous, mobile aliphatic carbons, especially paraffinic carbons, showed more linear isotherms than the others. The $n$ values (Table 4) ranged from 0.860 to 0.955 for NAP and from 0.828 to 0.969 for PHE while they equaled one for PYR, demonstrating sorption nonlinearity for NAP and PHE to some extent, and sorption linearity for PYR. Lin- ear sorption indicates that partitioning is the dominant mechanism for PYR sorption on POMs, possibly due to its higher hydrophobicity and larger molecular volumes which can result in a sorption mainly via solid-phase dissolution.

The organic carbon-normalized sorption capacity $\left(K_{\mathrm{oc}}\right)$ can be an indicator of sorption affinity for POM toward NAP, PHE and PYR. The $K_{\mathrm{oc}}$ value can be obtained from $K_{\mathrm{oc}}=K_{\mathrm{f}}^{\prime} / f_{\mathrm{oc}}$ [23]. As demonstrated in the present study, the $K_{\mathrm{oc}}$ value increased with increasing hydrophobicity of the sorbates, which is consistent with the results of literature [22]. For a given sorbate, the $K_{\text {oc }}$ values for POM1 were higher than the corresponding values for POM2, POM3 and POM4 (the $K_{\text {oc }}$ values of these three sorbents were similar). This observation together with the larger values of $n(>0.955)$ indicates that POM1 is a more powerful partition medium probably due to its lowest polarity and highest aliphaticity. The similar $K_{\mathrm{oc}}$ values for POM2, POM3 and POM4 are mainly due to their similar composition and configuration. The configuration of organic adsorbents is very important for the sorption of HOCs [28,29]. Xing [30] reported that the $K_{\mathrm{oc}}$ values were well correlated with the proportion of aromatic carbon, aliphatic carbon, aliphaticity and polar organic carbon. However, for a given POM, the sorption capacity $\left(K_{\mathrm{oc}}\right)$ increased in an order of PYR $>$ PHE $>$ NAP, illustrating sorption parameters are also related to the characteristics of sorbates. POM would have a higher sorption capacity when the sorbate has a lower solubility or a bigger octanol-water partition coefficient $\left(K_{\text {ow }}\right)$ value.

Polarity is an important parameter to regulate the sorption activity of organic pollutants. Kang and Xing [31] indicated that aliphatic-rich SOM with less polarity had a relative high $K_{\mathrm{oc}}$ value. It was also reported that diagenetically altered aromatic-rich SOM having relatively high sorption capacity $\left(K_{\mathrm{oc}}\right)$ was often associated with low polar functional group content or low polarity $[22,32]$. The sorption capacity of humic acids relates to their polarity. The lower the polarity is, the stronger the ability of humic acids towards hydrophobic organic pollutants is $[33,34]$. Similarly we found in this study that the highest sorption capacity $\left(K_{\mathrm{oc}}\right)$ of PAHs is correlated with the lowest polarity of the POM.

Fig. 3 gives the desorption isotherms of NAP and PHE. No significant desorption hysteresis was observed. The possible reasons for such desorption behaviors of PAHs in POMs may be as follows. First, the content of aliphatic carbon in POMs was much higher than that of aromatic carbon, which demonstrated that POMs were mainly composed of an expanded matrix in which a reversible sorption mechanism dominated [23]. Second, desorption behavior was also affected by polarity. Higher polarity may weaken the interactions between the adsorbents and PAH molecules [23,35], which contributed to the easy release of PAH molecules from the POMs.

Table 4

Modified Freundlich model parameters for PAH sorption on the POMs ${ }^{\mathrm{a}}$.

\begin{tabular}{|c|c|c|c|c|c|c|}
\hline PAHs & Sorbent & $K_{\mathrm{f}}^{\prime}$ & $n$ & $r^{2}$ & $f_{\mathrm{oc}}(\%)$ & $K_{\mathrm{oc}}$ \\
\hline \multirow[t]{4}{*}{ NAP } & POM1 & $27,210(1239)$ & $0.955(0.018)$ & 0.998 & 34.70 & 78,415 \\
\hline & POM2 & $15,254(1357)$ & $0.860(0.035)$ & 0.992 & 36.57 & 41,712 \\
\hline & РОМ3 & $16,703(834)$ & $0.897(0.020)$ & 0.998 & 36.05 & 46,333 \\
\hline & POM4 & $15,883(1574)$ & $0.907(0.041)$ & 0.994 & 31.24 & 50,842 \\
\hline \multirow[t]{4}{*}{ PHE } & POM1 & $36,206(3098)$ & $0.969(0.027)$ & 0.996 & 34.70 & 104,340 \\
\hline & POM2 & 17,085 (994) & $0.828(0.020)$ & 0.997 & 36.57 & 46,719 \\
\hline & РОМ3 & $17,405(1343)$ & $0.863(0.027)$ & 0.995 & 36.05 & 48,280 \\
\hline & POM4 & $22,749(1882)$ & $0.920(0.027)$ & 0.996 & 31.24 & 76,021 \\
\hline \multirow[t]{4}{*}{ PYR } & POM1 & $41,300(0)$ & $1(0.000)$ & 1 & 34.70 & 119,020 \\
\hline & POM2 & $27,533(0)$ & $1(0.000)$ & 1 & 36.57 & 75,288 \\
\hline & POM3 & $27,533(0)$ & $1(0.000)$ & 1 & 36.05 & 76,374 \\
\hline & POM4 & $27,533(0)$ & $1(0.000)$ & 1 & 31.24 & 88,134 \\
\hline
\end{tabular}

The data in parentheses are the standard error.

${ }^{a} K_{\mathrm{f}}^{\prime}$, a modified Freundlich parameter, was calculated after normalizing $C_{\mathrm{e}}$ by the supercooled liquid-state solubility $\left(S_{\mathrm{s}}\right)$ of PAHs, $q_{\mathrm{e}}=K_{\mathrm{f}}^{\prime}\left(C_{\mathrm{e}} / S_{\mathrm{s}}\right)^{n}$. The unit of $K_{\mathrm{f}}^{\prime}$ is mg/kg [19]; $f_{o c}$, the fraction of carbon in the POMs; $K_{\text {oc }}$, distribution coefficients. 

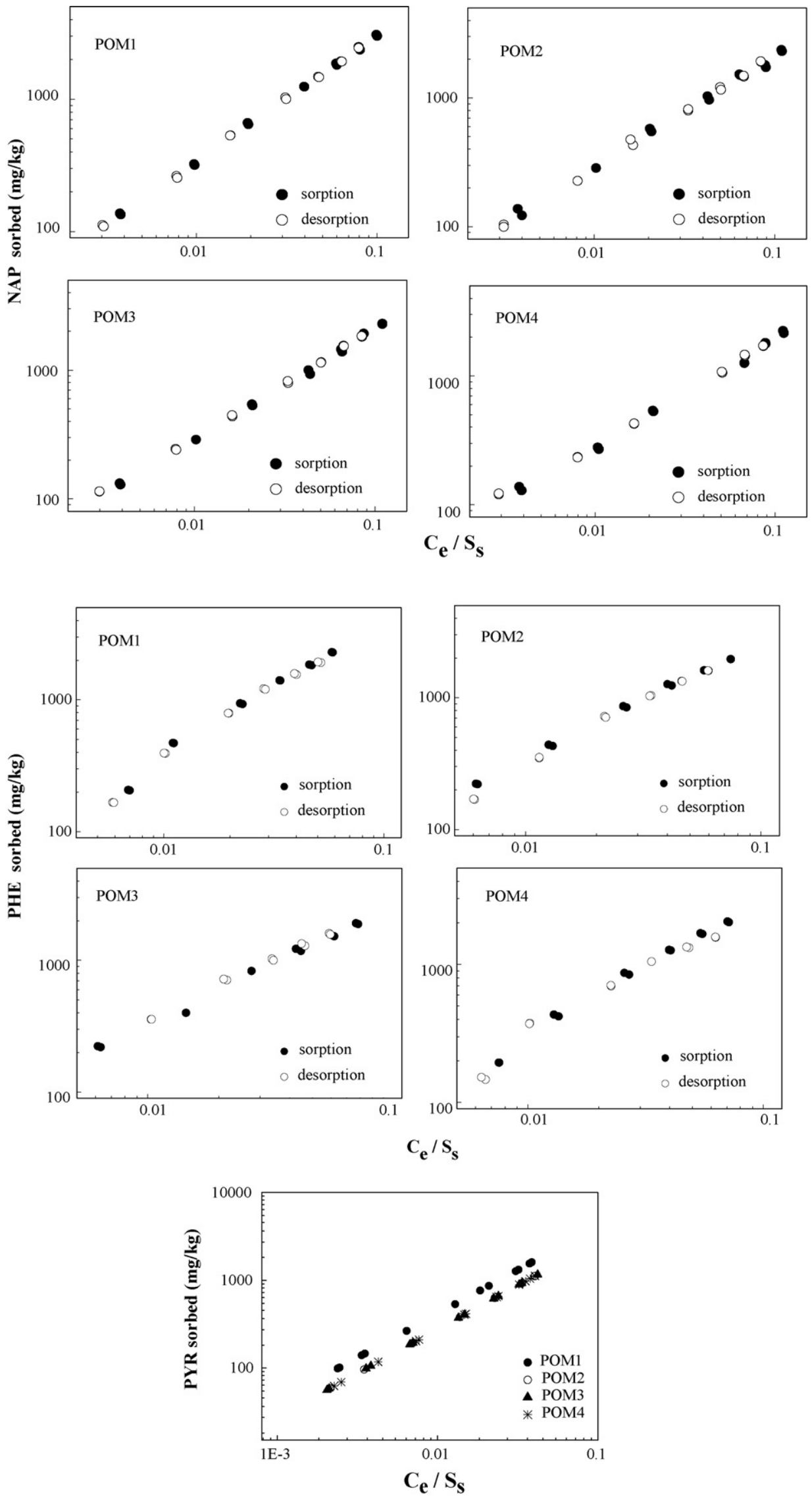

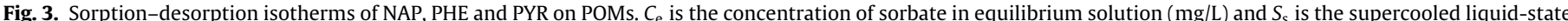
solubility (mg/L). 


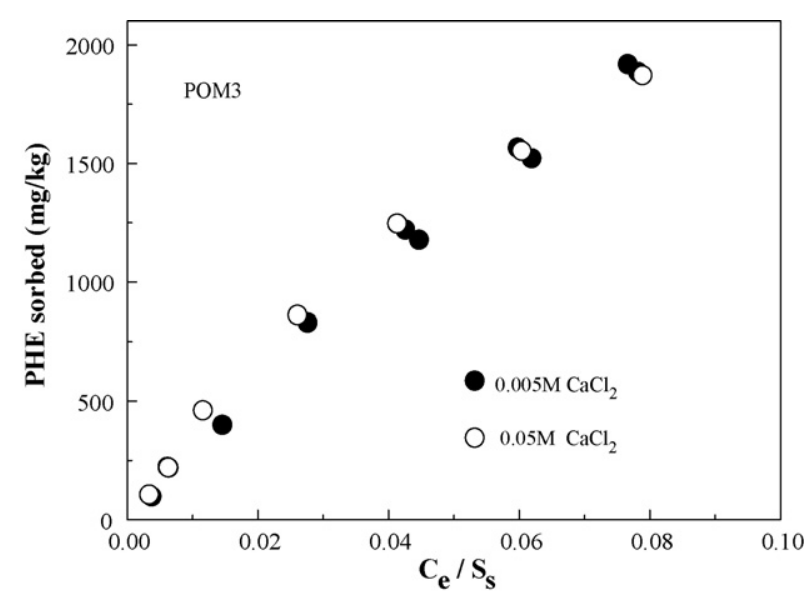

Fig. 4. Effect of ionic strength on the sorption of PHE by POM3. $C_{\mathrm{e}}$ is the concentration of sorbate in equilibrium solution $(\mathrm{mg} / \mathrm{L})$ and $S_{\mathrm{s}}$ is the supercooled liquid-state solubility $(\mathrm{mg} / \mathrm{L})$.

\subsection{Effect of ionic strength}

As can be found in Fig. 4, there is no significant difference between $\mathrm{CaCl}_{2}$ concentration of 0.005 and $0.05 \mathrm{~mol} / \mathrm{L}$ on the sorption of PHE on POM3. Ionic strength can affect the configuration of organic matter and influence the sorption of HOCs. Murphy et al. [36] reported that humic acids easily formed "open" or "linear" configuration at low ionic strength, which showed a high sorption capacity of HOCs. On the contrary, at high ionic strength, humic acids existed as a "coiled" configuration, resulting in the decrease of HOC sorption capacity. Lee et al. [37] demonstrated that different types of electrolytes had different effects on the interactions between humic acids and PAHs, and that the differences were primarily caused by the type of cations, not by the anions. Luo et al. [38] indicated that metal cations posed facilitating effects on PHE sorption in soils. The cations in the background electrolyte can be bound to the functional groups of the organic matter such as carboxyl and phenolic functionality, which greatly decreases the repulsion of the functional groups of organic matter, resulting in the formation of "close" or "coiled" configuration of polymer. Therefore, possible explanation for the lack of the effect of ionic strength on PHE sorption on the POM in this study could be (1) low water-solubility of POM avoided the transformation of the structure and configuration of POM from condensation or flocculation with increasing $\mathrm{CaCl}_{2}$ concentration, therefore, no obvious changes in PHE sorption was found; (2) PHE was primarily sorbed via solid-phase dissolution, and such sorption was almost not affected by ionic strength.

\section{Conclusions}

This study demonstrates that POMs have similar composition and configuration and are mainly composed of aliphatic components with higher polarity. Therefore, similar sorption capacities for these PAHs among the POMs was observed in this study. Composition of aliphatic components with higher polarity is responsible for the limited nonlinear sorption of NAP and PHE and a linear sorption of PYR on POM. The approximately linear sorption for NAP and PHE and linear sorption for PYR on POMs may suggest that partition is the dominant mechanism for PAH sorption on POM. This sorption behavior of POM is different from that of humic fractions such as humin and can be attributed to the shorter residence time in soils and less humified nature of POM. Therefore, POM may play an important function in short-term sorption of HOCs after they enter soils. Redistribution of HOCs among organic matter fractions such as from POM to humins may occur later. Turnover of POM may also influence sorption and desorption of HOCs in soils. These behaviors of HOCs in soil are of great significance in determining their fate in soils and need further study.

\section{Acknowledgements}

This work was funded by the National Natural Science Foundation of China (Projects 40730740, 20890110 and 20621703).

\section{References}

[1] C.T. Chiou, Partition and Adsorption of Organic Contaminants in Environmental Systems, Wiley Interscience, Hoboken, New Jersey, 2002.

[2] B. Pan, P. Ning, B. Xing, Part IV-sorption of hydrophobic organic contaminants, Environ. Sci. Pollut. Res. Int. 15 (2008) 554-564.

[3] J.J. Pignatello, B. Xing, Mechanisms of slow sorption of organic chemicals to natural particles, Environ. Sci. Technol. 30 (1996) 1-11.

[4] M.J. Salloum, B. Chefetz, P.G. Hatcher, Phenanthrene sorption by aliphatic-rich natural organic matter, Environ. Sci. Technol. 36 (2002) 1953-1958.

[5] M.T.O. Jonker, A.A. Koelmans, Polyoxymethylene solid phase extraction as a partitioning method for hydrophobic organic chemicals in sediment and soot, Environ. Sci. Technol. 35 (2001) 3742-3748.

[6] B.L. Chen, E.J. Johnson, B. Chefetz, L.Z. Zhu, B.S. Xing, Sorption of polar and nonpolar aromatic organic contaminants by plant cuticular materials: role of polarity and accessibility, Environ. Sci. Technol. 39 (2005) 6138-6146.

[7] M.T.O. Jonker, Absorption of polycyclic aromatic hydrocarbons to cellulose, Chemosphere 70 (2008) 778-782.

[8] J. Zhang, M. He, Y. Shi, Comparative sorption of benzo[ $\alpha$ ]pyrene to different humic acids and humin in sediments, J. Hazard. Mater. 166 (2009) 802-809.

[9] E. Besnard, C. Chenu, J. Balesdent, P. Puget, D. Arrouays, Fate of particulate organic matter in soil aggregates during cultivation, Eur. J. Soil Sci. 47 (1996) 495-503.

[10] E.T. Elliott, Rationale for developing bioindicators of soil health, in: C.E. Pankhurst, B.M. Doube, V.V.S.R. Gupta (Eds.), Biological Indicators of Soil Health, Commonwealth Agricultural Bureau, International, Wallingford, Oxon, UK, 1997, pp. 49-78.

[11] M.K. Zhang, Z.X. Ke, Copper and zinc enrichment in different size of particulate organic matter from polluted soils, Pedosphere 14 (2004) 27-36.

[12] M. Balabane, F. Van Oort, Metal enrichment of particulate organic matter in arable soils with low metal contamination, Soil Biol. Biochem. 34 (2002) 1513-1516.

[13] X.Y.Guo, S.Z.Z Zhang, X.Q. Shan, L. Luo, Z.G. Pei, T. Liu, Y.N. Xie, Characterization of $\mathrm{Pb}, \mathrm{Cu}$ and $\mathrm{Cd}$ adsorption on particulate organic matter in soil, Environ. Toxicol. Chem. 25 (2006) 2366-2373.

[14] J. Sebastia, F. Van Oort, I. Lamy, Buffer capacity and Cu affinity of soil particulate organic matter (POM) size fractions, Eur. J. Soil. Sci. 59 (2008) 304-314.

[15] P. Benoit, I. Madrigal, C.M. Preston, C. Chenu, E. Barriuso, Sorption and desorption of non-ionic herbicides onto particulate organic matter from surface soils under different land uses, Eur. J. Soil Sci. 59 (2008) 178-189.

[16] L.J. Krutz, S.A. Senseman, R.M. Zablotowicz, M.A. Matocha, Reducing herbicide runoff from agricultural fields with vegetative filter strips: a review, Weed Sci. 53 (2005) 353-367.

[17] J. Ducaroir, I. Lamy, Evidence of trace metal association with soil organic matter using particle size fractionation after physical dispersion treatment, Analyst 120 (1995) 741-745.

[18] W.L. Huang, W.J. Weber Jr., A distributed reactivity model for sorption by soils and sediments. 10. Relationships between desorption, hysteresis, and the chemical characteristics of organic domains, Environ. Sci. Technol. 31 (1997) 2562-2569.

[19] A.M. Carmo, L.S. Hundal, M.L. Thompson, Sorption of hydrophobic organic compounds by soil materials: application of unit equivalent Freundlich coefficients, Environ. Sci. Technol. 34 (2000) 4363-4369.

[20] S. Marinari, K. Liburdi, G. Masciandaro, B. Ceccanti, S. Grego, Humificationmineralization pyrolytic indices and carbon fractions of soil under organic and conventional management in central Italy, Soil Till. Res. 92 (2007) 10-17.

[21] B.T. Christensen, Physical fractionation of soil and structural and functional complexity in organic matter turnover, Eur. J. Soil. Sci. 52 (2001) 345-353.

[22] B. Xing, The effect of the quality of soil organic matter on sorption of naphthalene, Chemosphere 35 (1997) 633-642.

[23] L.Y. Huang, T.B. Boving, B.S. Xing, Sorption of PAHs by aspen wood fibers as affected by chemical alterations, Environ. Sci. Technol. 40 (2006) 3279-3284.

[24] X.L. Wang, B.S. Xing, sorption of organic contaminants by biopolymer-derived chars, Environ. Sci. Technol. 41 (2007) 8342-8348.

[25] C. Liang, Z. Dang, B. Xiao, W. Huang, C. Liu, Equilibrium sorption of phenanthrene by soil humic acids, Chemosphere 63 (2006) 1961-1968.

[26] B. Wen, J.J. Zhang, S.Z. Zhang, X.Q. Shan, S.U. Khan, B.S. Xing, Phenanthrene sorption to soil humic acid and humin fractions, Environ. Sci. Technol. 41 (2007) 3165-3171.

[27] J.W. Weber Jr., P.M. McGinley, L.E. Katz, A distributed reactivity model for sorption by soils and sediments. 1 . Conceptual basis and equilibrium assessments, Environ. Sci. Technol. 26 (1992) 1955-1962.

[28] X.L. Wang, R. Cook, S. Tao, B.S. Xing, Sorption of organic contaminants by biopolymers: role of polarity, structure and domain spatial arrangement, Chemosphere 66 (2007) 1476-1484. 
[29] J.L. Bonin, M.J. Simpson, Variation in phenanthrene sorption coefficients with soil organic matter fractionation: the result of structure or conformation? Environ. Sci. Technol. 41 (2007) 153-159.

[30] B. Xing, Sorption of anthropogenic organic compounds by soil organic matter: a mechanistic consideration, Can. J. Soil Sci. 18 (2001) 317-323.

[31] S. Kang, B.S. Xing, Phenanthrene sorption to sequentially extracted soil humic acids and humins, Environ. Sci. Technol. 39 (2005) 134-140.

[32] M.D. Johnson, W. Huang, W.J. Weber Jr., A distributed reactivity model for sorption by soils and sediments. 13. Simulated diagenesis of natural sediment organic matter and its impact on sorption/desorption equilibria, Environ. Sci. Technol. 35 (2001) 1680-1687.

[33] C.T. Chiou, R.L. Malcolm, T.I. Brinton, D.E. Kile, Water solubility enhancement of some organic pollutants and pesticides by dissolved humic and fulvic acids, Environ. Sci. Technol. 20 (1986) 502-508.
[34] C.T. Chiou, D.E. Kile, Deviations from sorption linearity on soils of polar and nonpolar organic compounds at low relative concentrations, Environ. Sci. Technol. 32 (1998) 338-343.

[35] B. Chefetz, A.P. Deshmukh, P.G. Hatcher, Pyrene sorption by natural organic matter, Environ. Sci. Technol. 34 (2000) 2925-2930.

[36] E.M. Murphy, J.M. Zachara, S.C. Smith, J.L. Phillips, T.W. Wietsma, Interaction of hydrophobic organic compounds with mineral-bound humic substances, Environ. Sci. Technol. 28 (1994) 1291-1299.

[37] C.L. Lee, L.J. Kuo, H.L. Wang, P.C. Hsieh, Effects of ionic strength on the binding of phenanthrene and pyrene to humic substances: three-stage variation model Water Res. 37 (2003) 4250-4258.

[38] L. Luo, S.Z. Zhang, Y.B. Ma, P. Christie, H.L. Huang, Facilitating effects of metal cations on phenanthrene sorption in soils, Environ. Sci. Technol. 42 (2008) 2414-2419. 